3. The use of specific reagents to block different groups on the protein molecule, has shown that either a phenolic or an amino group on the protein may be concerned in its combination with the vitamin. The point at which the protein attaches itself to the cyanocobalamin molecule is also discussed.

We are indebted to Dr R. A. Kekwick of the Lister Institute for the gift of $2^{\prime}$-acetylthioethylacetamide and to Dr M. Dixon, F.R.S., of the School of Biochemistry, Cambridge, for the $p$-chloromercuribenzoate. In the experiments using $\left[{ }^{14} \mathrm{CN}\right]$ cobalamin, the measurements of radioactivity were done by Dr R. F. Glascock, to whom we express our thanks. We also wish to thank Dr S. K. Kon for his interest in this work and valuable help in preparing it for publication. This paper forms part of a Ph.D. thesis presented by one of us (M. E. G.) to the University of Reading.

\section{REFERENCES}

Armitage, J. B., Cannon, J. R., Johnson, A. W., Parker, L. F. J., Smith, E. L., Stafford, W. H. \& Todd, A. R. (1953). J. chem. Soc. p. 3849.

Baddiley, J., Kekwick, R. A. \& Thain, E. M. (1952). Nature, Lond. 170, 968.

Bolling, D., Sober, H. A. \& Block, R. J. (1949). Fed. Proc. 8, 185.
Boxer, G. E. \& Rickards, J. C. (1951). Arch. Biochem. 30, 372.

Brown, F. B. \& Smith, E. L. (1954). Biochem. J. 56, xxxiv. Calvery, H. O., Herriott, R. M. \& Northrop, J. H. (1936). J. biol. Chem. 113, 11.

Elson, L. A. \& Morgan, W.T.J.(1933). Biochem. J.27, 1824.

Gant, D. E., Smith, E. L. \& Parker, L. F. J. (1954). Biochem. J. 56, xxxiv.

Glascock, R. F. (1954). Isotopic Gas Analysis for Biochemists, chs. III and Iv. New York: Academy Press Inc. Gregory, M. E. (1954). Brit. J. Nutr. 8, 340.

Gregory, M. E. \& Holdsworth, E. S. (1953). Biochem. J. 55, 830.

Gregory, M. E. \& Holdsworth, E. S. (1955). Biochem. J. 59, 329.

Grunbaum, B. W., Schaffer, F. L. \& Kirk, P. L. (1952). Analyt. Chem. 24, 1487.

Holiday, E. R. (1936). Biochem. J. 30, 1795.

Morris, D. L. (1948). Science, 107, 254.

Olcott, H. S. \& Fraenkel-Conrat, H. (1947). Chem. Rev. 41, 151.

Partridge, S. M. (1949). Nature, Lond., 164, 443.

Pfiffner, J. J., Dion, H. W. \& Calkins, D. G. (1952). Fed. Proc. 11, 269.

Pitney, W. R., Beard, M. F. \& Van Loon, E. J. (1954). J. biol. Chem. 207, 143.

Toennies, G. \& Kolb, J. J. (1951). Analyt. Chem. 23, 823.

Wijmenga, H. G., Thompson, K. W., Stern, K. G. \& O'Connell, D. J. (1954). Biochim. biophys. Acta, 13, 144.

\title{
An Apparatus for Continuous Electrophoresis on Paper
}

\author{
By E. S. HOLDSWORTH \\ National Institute for Research in Dairying, University of Reading
}

(Received 24 July 1954)

The application of electrophoresis in solution with paper as a supporting medium, for separating and detecting substances which have different mobilities in an electric field is now well established. The technique to be described differs from others in that the mixture to be separated is continuously fed into the apparatus and the separated components can be continuously removed, i.e. it can be used as a preparative method. An attempt was made by Philpot (1940) to use electrophoresis in a thin horizontal layer of solution between layers of electrolyte, as a continuous process. Some of the difficulties of this technique were overcome by Svensson \& Brattsten (1949), by using a thin vertical layer and powdered glass to prevent mixing during the separation. There was a constant flow of buffer solution down through the glass and out through a series of tubes at the bottom of a narrow Perspex box. If a constant supply of material was applied at one place on the top surface it was carried down by the buffer solution to the outlet immediately below.
However, if a potential gradient was applied at the ends of the box the substance was deflected towards one electrode and appeared at some other outlet, depending on its rate of movement in the electrical field. With a mixture of substances of different mobilities a continuous separation could be effected. The apparatus seems to have found very little practical application, probably owing to the difficulty of setting up and also to the poor resolution obtainable. These workers also tried a sheet of filter paper as a supporting medium for the electrolyte but with little success. Grassmann \& Hannig (1950) were the first to use paper successfully in this type of apparatus. Platinum wire electrodes were fixed to the sides of the paper and the buffer solution was allowed to drip off at a series of points. Successful continuous separations of amino acid mixtures and proteins were reported. A forerunner of this type of apparatus was that of Haugaard \& Kroner (1948), who carried out paper chromatography on a spot of the material to be separated and applied an electric 
field across the paper, at right angles to the flow of solvent. Separation was achieved by two separate processes, chromatography in the downward direction and electrophoresis horizontally, but the process in this technique was discontinuous. A slightly different type of apparatus was described by Durrum (1951), who obtained continuous separation of dyestuffs, amino acid mixtures and serum proteins. Strain \& Sullivan (1951) have used this method for inorganic preparative work. An apparatus incorporating improvements but based on these previous models was demonstrated to the Biochemical Society (Holdsworth, 1953), and has been successfully used for the preparation of pure milk proteins (cf. Gregory \& Holdsworth, 1955). A recent publication of Grassmann \& Hannig (1953) has dealt comprehensively with the theory and practice of this technique. Although simple in principle the successful continuous operation of this method must overcome numerous technical diffculties, and the way in which many of these have been overcome in this laboratory is described in this paper.

\section{PRINCIPLE}

When the paper is acting solely as an inactive supporting medium, the angle to the vertical of the path taken by a particular component is $\alpha$, where

$$
\begin{aligned}
& \tan \alpha=\frac{\text { Rate of movement in the electrical field }}{\text { Rate of movement down the paper (i.e. }} \\
& \text { of the buffer solution) }
\end{aligned}
$$

It is evident that for this method to be successful it is essential to have close control over all the variables which will be considered in turn.

(i) The electrical field strength must be constant. The application of the field to only the bottom corners of the paper (Durrum, 1951) even when this has extra thicknesses at the edges (Holdsworth, 1953) means that the potential difference between the two sides steadily diminishes as the top of the paper is approached. For long periods of operation it is convenient to have a uniform field, therefore the electrodes must extend down the edges of the paper (cf. Grassmann \& Hannig, 1953).

(ii) The rate of flow of the electrolyte down the paper and its composition must be kept constant. The supply of electrolyte is usually provided by allowing the paper to dip into a trough of electrolyte supplied with a constant-level device. On its passage down the paper the electrolyte is subjected to the heating effect of the current, which causes evaporation of the water. Thus non-volatile solutes, including buffer salts become more concentrated in their progress down the paper. For many purposes it is possible with a sufficiently rapid rate of flow to achieve a steady state; the only disadvantage of this concentrating effect then is that the path followed by a substance in the electrical field is nonlinear. By cooling the paper (as in the apparatus to be described) this effect is eliminated and heatlabile materials are not destroyed.

(iii) The products of electrolysis must not be allowed to bring about a change of $\mathrm{pH}$ on the paper. The removal of these products by arranging an extra flow of buffer down the edges of the paper (Holdsworth, 1953; Grassmann \& Hannig, 1953) is not entirely satisfactory and in the apparatus described below the electrodes are continually washed with fresh buffer solution and are separated from the paper by a cellophan membrane.

(iv) The methods described so far have made no attempt to control the temperature of the system, reliance has been on the achievement of a steady state when the external temperature was kept constant. Even with low current densities the use of the high voltages necessary for separation results in significant increases of temperature in the apparatus, and therefore it was deemed advisable to remove the heat produced on the paper, in addition to having a constant external temperature.

\section{DESCRIPTION OF THE APPARATUS}

A general view of the apparatus is shown in the photograph (Fig. 1), and Figs. 2 and 3 show the constructional details. The apparatus is made of $\downarrow$ in. Perspex sheet and consists essentially of a framework $(A)$ which, when the removable back, top and two front panels $(B)$ are in position, forms the moisture-saturated enclosure for the paper. Any moisture collecting on the panels is conducted away by the gutters $(D)$.

The cooling system $(T)$ is a Perspex box with the front face $\frac{1}{16}$ in. thick. The whole of this face is cooled by water entering at $V$, passing round a series of baffles and eventually leaving at $W$. The Perspex cooler is attached by four stainless steel bolts to the central strut of the framework $C$, but separated from the strut by spacing units $(U)$ of Perspex. The two spacers at the bottom are in. thick; those at the top $\frac{9}{16}$ in. thick. This slight tilt of the cooler from the vertical ensures that the paper clings to the entire surface.

The trough $E$ measures $18 \times 2 \times 1 \frac{1}{2}$ in. high (internal) and along the top of one face is cemented a $\downarrow$ in. diameter Perspex rod $\left(F^{\prime}\right)$ over which the paper hangs. A constant head of electrolyte is ensured by a continual drip from the feed $(P)$ into the trough, the excess passing out of the overflow $(G)$ into a tank $(H)$ of capacity about 21 . A pump returns this buffer to the supply vessel, which is a 51 . aspirator bottle. Thesolution drips off the paper at 29 points into small glass funnels $(K)$ constructed from Pyrex tubing of internal diameters 9 and $3 \mathrm{~mm}$. The centres of these funnels are $\frac{1}{2}$ in. apart and are held in a Perspex rack $(L)$. Fractions are collected either in $150 \times 9 \mathrm{~mm}$. test tubes or into larger vessels. By removing the lower front panel a rack of test tubes $(N)$ may be replaced by fresh tubes daily, without. disturbing the paper, since the lower part of the funnels $(K)$ consists of lengths of flexible polyvinyl chloride (P.v.c.) tubing. The first and last drip points (see Fig. 4) serve a wide strip of paper, and the liquid is collected in correspondingly larger vessels. 
Support for the electrodes is provided by strips of Perspex $17 \times 1 \times \frac{1}{4}$ in. which have a U-shaped channel milled out $\frac{1}{8}$ in. deep, $\frac{3}{8}$ in. across and $\frac{1}{8}$ in. from one edge $(I)$. The electrodes are $20 \mathrm{in}$. lengths of $0.38 \mathrm{~mm}$. platinum wire $(S)$ enclosed in a length (approx. 16 in.) of cellophan tubing $(Q)$ ( $\frac{8}{32}$ in., Visking Corporation). The bottom of the cellophan tubing is tightly bound with linen thread over a 2 in. length of $6 \mathrm{~mm}$. diameter glass tube covered with flexible P.v.c. tubing and is connected to the outlet of a Perspex reservoir $(J) 3 \times 2 \frac{1}{2} \times 3$ in., placed on the same shelf which supports the trough $E$. The top end of the cellophan tube is fastened, in the same way as the lower end to the glass connexion $(R)$ (Fig. 3) made from $6 \mathrm{~mm}$. Pyrex tubing. This construction is necessary to allow for the introduction of the platinum electrodes $(S)$, for the escape of gases produced by electrolysis and for the overflow of electrolyte. The cellophan tubing is fastened to the Perspex supports $(I)$ where they are stiffened by the underlying glass connecting tubes by clips made from $\frac{1}{8}$ in. diameter stainless-steel rod covered with P.v.c. tube. A diagram of this flowing electrode system is shown in Fig. 3. The flattened, circular centre portion of $R$ is displaced slightly backwards from the plane of the cellophan tubing and the paper, so as to afford no obstruction to the 'hang' of the paper from the trough. The overflow from anode and cathode is taken to the Perspex tank $H$, where it mixes and is returned to the aspirator bottle by the pump.
It is necessary to discharge the two overflow tubes into the top of the tank $H$ well above the level of the electrolyte to eliminate the possibility of a short circuit. To ensure a good contact between the paper and the cellophan tubing, strips of Perspex $15 \times \frac{8}{8} \times \frac{1}{4}$ in. press the paper against the electrodes and are kept in place by stainless steel clips. Electrolyte is added dropwise, to prevent short circuiting, to the reservoir vessels $J$. In this way a continuous change of liquid surrounding the platinum wire is obtained. Furthermore, the paper is separated from the electrodes by a cellophan membrane, which ensures that $\mathrm{pH}$ changes do not affect the paper.

The paper is cut from a piece of $18 t \times 22 \frac{1}{2}$ in. filter paper to the dimensions shown in the diagram (Fig. 4). The 3 in. portion at the top is doubled back to dip into the trough and is kept in position by a thick glass rod. The surface of the cooler $(T)$ is treated with Silicone M.S. 550 to make it waterrepellent. The electrolyte is sprayed on the paper, which is pressed against the surface of the cooler eliminating all air pockets. The paper points are then pushed into the glass funnels making sure that the points adhere to the glass by capillary attraction, since only with a good contact can there be an even flow of electrolyte from the paper. With trough $E$ full of electrolyte and the apparatus closed, steady state conditions of flow down the paper and saturation of the atmosphere are reached in about $4 \mathrm{hr}$.

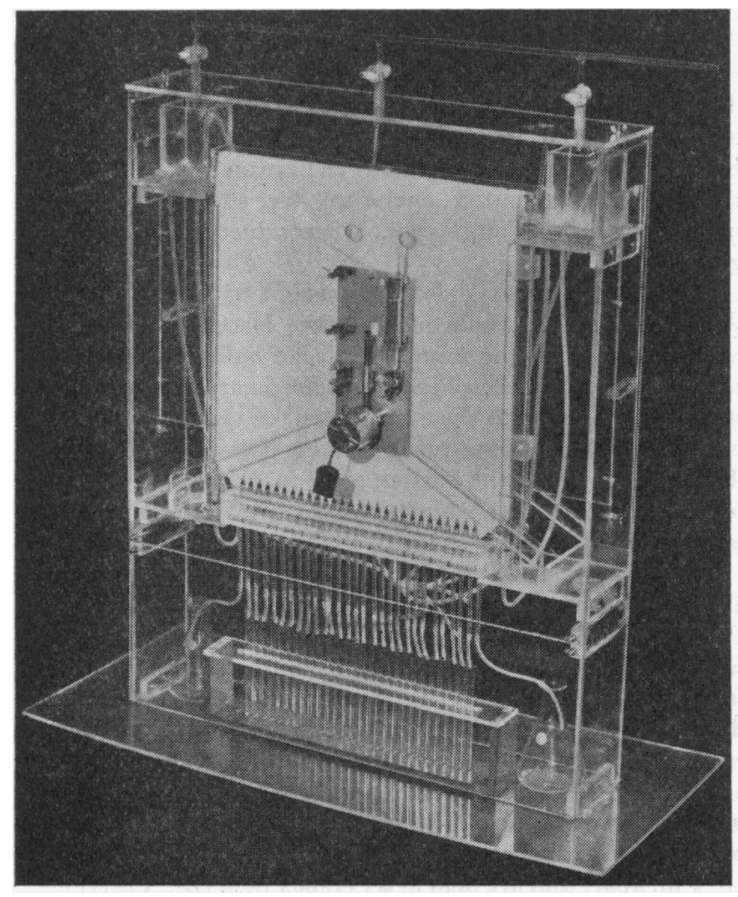

Fig. 1

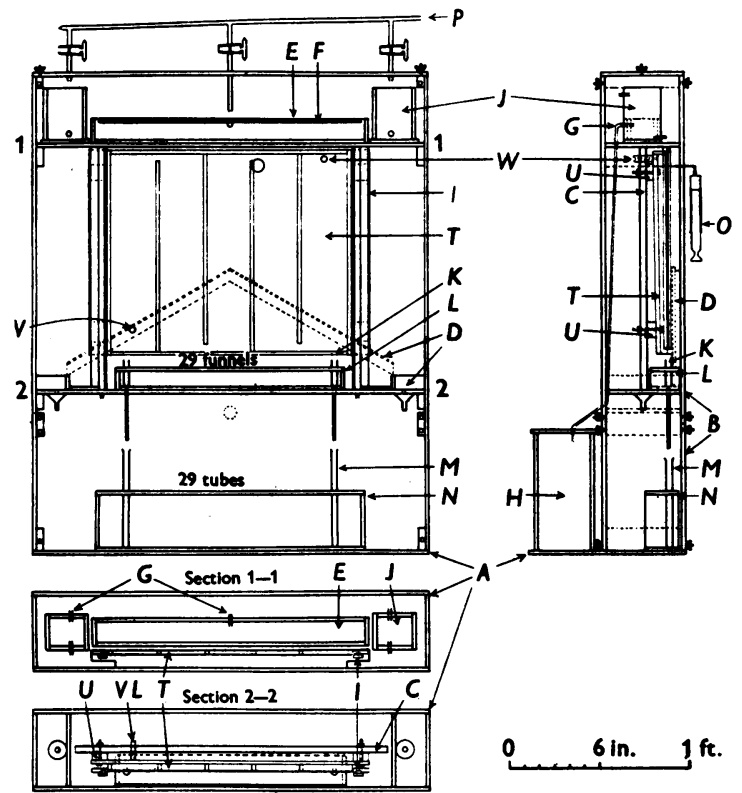

Fig. 2

Fig. 1. Apparatus for continuous electrophoresis on paper.

Fig. 2. Diagram of the apparatus for continuous electrophoresis on paper. $A$, frame; $B$, front panëls; $C$, strut; $D$, condensate collector; $E$, trough; $F$, rod; $G$, overflow; $H$, tank; $I$, electrode supports; $J$, reservoirs; $K$, funnels; $L$, funnel holder; $M$, tubes; $N$, tube holder; $O$, syringe; $P$, electrolyte feed; $T$, cooling system; $U$, spacing pieces; $V$, water inlet; $W$, water outlet. 
Temperature control. The apparatus is used in a room where the temperature is controlled at $20 \pm 1^{\circ}$. The cooling system for the paper is supplied from a large glass tank of water $20 \times 20 \times 10$ in. cooled by a glass coil fed from the cold tap. The temperature of this reservoir is then maintained at $19^{\circ}$ (i.e. $1^{\circ}$ less than ambient temperature) by a Circotherm pump and heater unit (Shandon Scientific Co.).

Method of applying the test solution. The usual method of applying material to be separated by a wick of filter paper $1 \mathrm{~mm}$. wide (Durrum, 1951; Grassmann \& Hannig, 1953) is not very suitable for long periods of operation and a mechanically operated syringe was made in the Engineering Department, of this Institute, by Mr E. J. Pinnegar. Experience has shown that the system can deal with material at the rate of 1-5 ml. per $24 \mathrm{hr}$. By using either a $20 \mathrm{ml}$. or a $10 \mathrm{ml}$. Chance's interchangeable all-glass syringe, amounts of 4 or $2 \mathrm{ml}$. can be delivered in $24 \mathrm{hr}$. by the arrangement, shown in Fig. 1.

Two glass syringes are selected, ejecting 2 and $4 \mathrm{ml}$. respectively for a plunger travel of $\mathbf{0 . 4 8 0}$ in., the barrels of which are attached by four 'Terry' spring clips to a brass base-plate $9 \times 4 \times 18$ in. s.w.g. Spring clips are also used to attach the plunger handles to the ends and at right angles to a carrier bar $3 \frac{3}{4} \times \frac{5}{16} \times \frac{1}{8}$ in., the centre of which is silversoldered at $90^{\circ}$ to a $\frac{5}{16}$ in. inside diameter brass carrier tube 11 in. long. Into this a $\frac{5}{16}$ in. outside diameter, 10 square threads/in. worm, $3 \frac{1}{2}$ in. long, is fitted. A spring-loaded pawl is fixed to the carrier tube to mesh with the worm and provides a linear drive to the syringe plunger. The worm is driven by a 2 rev./hr. Sangamo Weston motor unit through a worm-and-pinion gear, providing a speed reduction of $10: 1$. The reduction gear pinion is assembled on the carrier worm spindle, which is then mounted between two bearing brackets bent up from the base-plate, and the motor unit with the reduction worm pinned to the motor spindle is then screwed to the base-plate with reduction worm and pinion in mesh.

To fill the syringes it is necessary only to depress the spring-loaded pawl and draw the brass carrier tube down to the stops provided.

The chosen syringe is fitted with a glass right-angled needle, but to prevent the paper being pierced, the final contact between needle and paper is by a short length of flexible P.v.c. tubing, $0.5 \mathrm{~mm}$. bore. The brass plate is fastened to the front Perspex panel (Fig. 1) by two stainless steel 8 B.A. bolts, and a hole is bored through the panel for entry of the needle. Two positions, one central and the other one-third of the way from one edge of the paper are suitable for most requirements, particularly since the polarity of the electrodes can be reversed. The point of application of the material is $2 \mathrm{in}$. below the lip of the trough $E$.

Power supply. A source of d.c. voltage stabilized by three STV 780/40 glow discharge tubes in series (Benson, 1950) was used to provide voltages up to $840 \mathrm{v}$ in steps of $70 \mathrm{v}$. Change of load in the range 2-30 ma causes a change of less than $1 \%$ in the potential across the paper.

\section{OPERATION OF THE APPARATUS}

The point at which a component will drip off the paper is determined by the electrophoretic mobility, the composition of the electrolyte, the voltage across the paper and the rate of movement down the paper, which in turn depends on the grade of paper used.

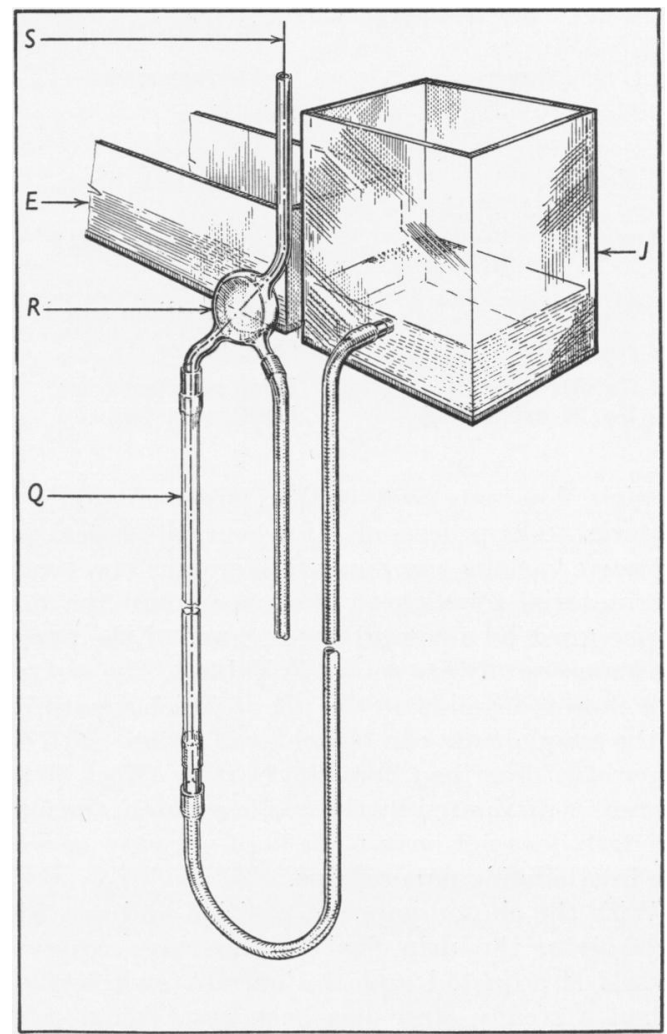

Fig. 3. Diagram of the flowing electrode system. $E, J$, as Fig. 2; $Q$, cellophan bag; $R$, flattened circular section; $S$, Pt wire.

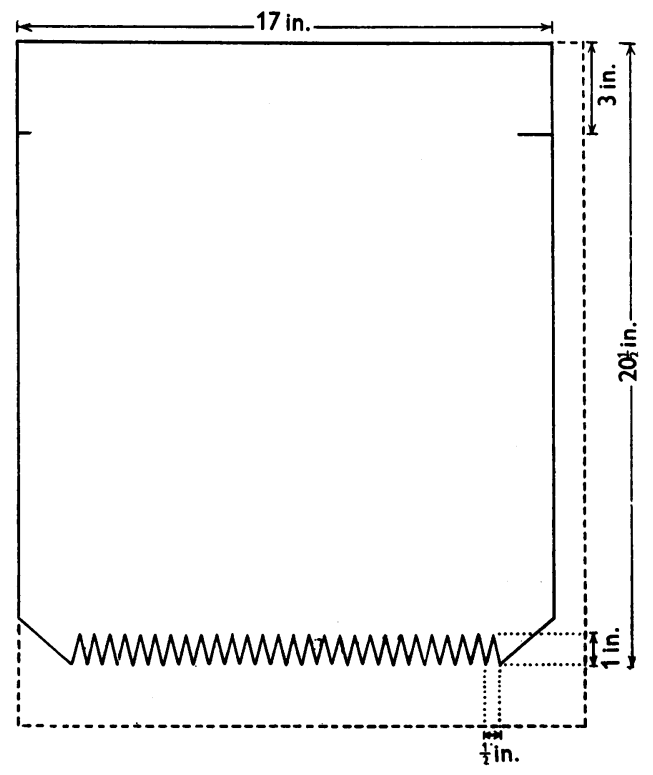

Fig. 4. Diagram showing dimensions of the paper for continuous electrophoresis. 
Table 1. Some Whatman papers suitable for continuous electrophoresis

\begin{tabular}{ll} 
No. 1 & \multicolumn{1}{c}{ Paper } \\
No. 2 & $\begin{array}{c}\text { Characteristics } \\
\text { Thin, slow }\end{array}$ \\
No. 54 & Medium-thick, slow \\
No. 3 MM & Thin, medium speed \\
No. 4 & Thick, medium speed \\
No. 31 & Thin, fast \\
No. 31 extra thick & Thin, very fast \\
\end{tabular}

A high flow rate will enable larger amounts of material to be processed. However, the separation between various components increases the longer the material remains on the paper, and the final choice must be a compromise. Some of the papers used successfully are shown in Table 1 . The electrolyte chosen depends on the $\mathrm{pH}$ at which separation of the components can be achieved. Since with the apparatus described here the heating effect of the current is dissipated by the cooling systen, the ionic strength does not have to be kept very low as with the free-hanging paper strips.

With the chosen paper in position and wet with electrolyte, the drip feed to the three reservoir vessels is adjusted and the current switched on. When a steady state has been established (after $4 \mathrm{hr}$.) the mechanically operated syringe is charged with a solution of the substance to be separated (dissolved in the electrolyte), fixed into position and the synchronous motor started. Where no adsorption takes place quantitative separations can be obtained by collecting fractions for several hours after all the contents of the syringe have been placed on the paper. However, it is often convenient to stop the process before the material has been completely washed off the paper, which is dried and stained to serve as a guide to the required fractions.

\section{CONCLUSIONS}

The technique of one-dimensional electrophoresis on paper has been employed for the detection of small quantities of a wide range of compounds, but is inconvenient for the preparation of larger amounts of material. The apparatus described in this article has been used for the continuous separation of mixtures in amounts up to $4 \mathrm{ml}$. of a $5 \%$ solution/ $24 \mathrm{hr}$. When the supporting medium is a thin sheet of close-textured paper, sharp resolution can be obtained. A little of this sharpness is lost by addition of the cooling system, but as this prevents accumulation of electrolyte owing to loss of water by evaporation, the position at which a substance drips off the paper is more stable over long periods of

\begin{abstract}
Remarks
For small quantities of substances of low mobility, e.g. some carbohydrates in borate buffers

As above, but capable of dealing with larger amounts of material

A tough paper very useful for small amounts of protein, e.g. enzymes, also for coenzymes or peptides

For the majority of protein separations

Can be used for proteins, peptides and sugars

For substances of high mobility, e.g. inorganic ions, organic acids and bases, dyestuffs, etc.
\end{abstract}

operation. When using paper from one batch, substances appear in the same tubes in experiments conducted at different times. The use of paper as a support has certain disadvantages. In protein separations, some proteins become strongly adsorbed on paper at $\mathrm{pH}$ values below 7 , thus limiting the range within which the process can be used. This difficulty could possibly be overcome by pretreatment of the paper, e.g. by esterification of carboxylic groups. There is no apparent reason why fabric made of fibres other than cellulose should not prove useful. Geldmacher-Mallinckrodt \& Weinland (1953) have already demonstrated that onedimensional separations of polysaccharides can be achieved by electrophoresis on closely woven silk.

The apparatus should be particularly useful for the further purification of materials when established procedures are cumbersome or inefficient. For instance, in the isolation of a cyanocobalaminprotein from milk a stage was reached when ammonium sulphate fractionation gave no further purification and the proteins contained only $1 \%$ of the required material. This mixture was readily resolved by continuous electrophoresis to give cyanocobalamin-protein free from the majority of the other proteins (see Fig. 4, Gregory \& Holdsworth, 1955).

\section{SUMMARY}

Details are given of the construction of an apparatus for continuous electrophoresis on paper. By means of a flowing electrode system long periods of operation can be used without $\mathrm{pH}$ change on the paper. Arrangements for cooling the paper allow the technique to be applied to heat labile materials (e.g. enzymes) and also lead to more reproducible results, since evaporation of solvent from the electrolyte is eliminated.

The polyvinyl chloride tubing was obtained from A. R. Horwell, 18 Christchurch Avenue, London, N.W. 6, Silicone fluid MS 550 from Hopkin and Williams, Chadwell Heath, Essex, the Sangamo motor unit, model 57, 2 rev./hr., was obtained from Sangamo-Weston, Enfield, Middlesex. The pump for returning electrolyte to the aspirator bottle was 
a Stuart-Turner pump in which the parts coming in contact with electrolyte were replaced by similar parts made of Perspex.

\section{REFERENCES}

Benson, F. A. (1950). Voltage Stabilizers, p. 23. London: Electronic Engineering.

Durrum, E. L. (1951). J. Amer. chem. Soc. 73, 4875.

Geldmacher-Mallinckrodt, M. \& Weinland, H. (1953). Hoppe-Seyl. Z. 292, 65.

Grassmann, W. \& Hannig, K. (1950). Naturwissenschaften, 37, 397.
Grassmann, W. \& Hannig, K. (1953). Hoppe-Seyl. Z. 292, 32.

Gregory, M. E. \& Holdsworth, E. S. (1955). Biochem. J. $59,329$.

Haugaard, G. \& Kroner, T. D. (1948). J. Amer. chem. Soc. $70,2135$.

Holdsworth, E. S. (1953). Biochem. J. 55, xiv.

Philpot, J. St L. (1940). Trans. Faraday Soc. 36, 38.

Strain, H. H. \& Sullivan, J. C. (1951). Analyt. Chem. 23, 816.

Svensson, H. \& Brattsten, J. (1949). Ark. Kemi, 1, 401.

\title{
Antithyroid Substances
}

\section{DERIVATIVES OF 2-MERCAPTOGLYOXALINES}

\author{
By A. LAWSON ANd C. E. SEARLE \\ Royal. Free Hospital School of Medicine, Hunter Street, London, W.C. 1
}

(Received 2 October 1954)

In Parts 1 and 2 (Searle, Lawson \& Hemmings, 1950; Searle, Lawson \& Morley, 1951) the examination of a range of 2-mercaptoglyoxalines (I), 2mercaptothiazoles and 2-thiohydantoins for antithyroid activity in young rats was described.

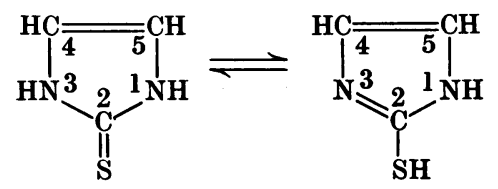

(I)

As a result of chemical investigations in the 2mercaptoglyoxaline series a number of new compounds have been obtained, and these have now been subjected to screening tests for antithyroid activity by the methods already described, namely by determining the effect of a single dose of the drug on the radioactive iodine uptake of the rat's thyroid gland over a $4 \mathrm{hr}$. period.

With a view to increasing the duration of activity of some antithyroid drugs, particularly the highly active 2-mercapto-1-methylglyoxaline ("methimazole', 'mercazole'), the effect of introducing a hydrolysable grouping on the sulphur atom has been investigated. Results obtained with one such compound, 2-ethoxycarbonylthio-1-methylglyoxaline ('carbimazole', 'neomercazole') (II, $\mathrm{R}=\mathrm{OC}_{2} \mathrm{H}_{5}$ ) were described by Lawson, Rimington \& Searle (1951). This substance is at least as active in the rat as its parent thiol, but no evidence was obtained that it was hydrolysed sufficiently slowly in vivo to give a markedly increased duration of activity. In clinical use (Doniach, 1953; Poate,
1953), however, it appears to be slightly more active than 2-mercapto-1-methylglyoxaline, and the substance is free of the bitter taste associated with the thiol drugs.

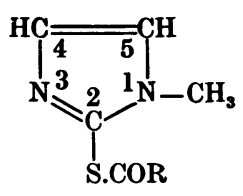

(II)

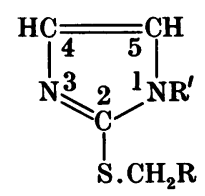

(III)
A preliminary account has appeared of the antithyroid activity of some further S-substituted derivatives of 2-mercaptoglyoxalines (Lawson \& Searle, 1954). Reaction of 2-mercapto-1-methylglyoxaline with methyl chloroformate, benzyl chloroformate or phosgene yields derivatives (II) which are readily hydrolysed in vitro back to the thiol, and which show high antithyroid activity in the rat. Reaction of mercaptoglyoxalines with alkyl halides, on the other hand, yields thioethers (III) which can only be hydrolysed back to the parent thiols under much more drastic conditions.

It is usually assumed that antithyroid agents such as thiourea and the thiouracils and mercaptoglyoxalines owe their activity to the presence of the potentially free thiol group, which by its ready reduction of elementary iodine to iodide ion

$$
2 \mathrm{R}-\mathrm{S}^{-}+\mathrm{I}_{2} \rightarrow \mathrm{R}-\mathrm{S}-\mathrm{S}-\mathrm{R}+2 \mathrm{I}^{-}
$$

interferes with the iodination of tyrosine in the thyroid protein and hence inhibits the synthesis of the thyroid hormone. 